\title{
Peculiarities of Swift Proton Transmission through Tapered Glass Capillaries
}

\author{
F.F. Komarov*, A.S. Kamyshan and V.V. PiL'Ko \\ Institute of Applied Physics Problems, Belarussian State University \\ 7 Kurchatov Str., 220118, Minsk, Belarus
}

\begin{abstract}
A study of the 150-300 keV proton beam transmission through glass (borosilicate) tapered capillaries with different diameters of the input and output of the capillary was performed. The focusing effect was observed. The areal density of the transmitted beam is enhanced by approximately 20 times. It was shown that changing a taper angle from $0.5 \mathrm{deg}$ to $1.7 \mathrm{deg}$ evidences the increase of the transmission coefficient more than by 300 times keeping the initial energy spectrum of ions.
\end{abstract}

PACS: $34.35 .+\mathrm{a}$

\section{Introduction}

Slightly tapered glass capillaries with micro or submicro outlet diameters attract much attention as ion beam focusing lens [1-4]. The smallest beam spot size obtained so far was $0.3 \mu \mathrm{m}[1]$. Previous studies have demonstrated the focusing effect with respect to many kinds of ions and energies [1-4]. The focusing factor of a glass capillary, defined as the ratio of areal current density at the inlet and outlet, depends on the ion species, the incident energies and the shape of glass capillary. This factor of $8 \mathrm{keV} \mathrm{Ar}^{8+}$ is about 10 and for $2 \mathrm{MeV} \mathrm{He}^{+}$ beam amounts to about 1000 [1-4].

The purpose of the present report is to study the dependence of proton transmission through capillaries on capillary taper angles. An ion energy region was chosen to satisfy the experimental conditions of local implantation, local elemental and structure analysis, micro- and nanolithography, X-ray radiography, and applications in biology and medicine $[3,5]$. For example, this technique enables in-air particle-induced X-ray emission (PIXE) measurements of various samples that are not compatible with the vacuum environment [2] (wet solids, liquids and gases). Slightly tapered glass capillary optics is applied to work as a differential pumping orifice as well as a focusing lens [2]. Recently, the processes of interaction of accelerated protons with the surface of cylindrical insulating capillaries as well as time and angular distributions of ions transmitted through such capillaries have been studied in our paper [6].

\section{Experimental technique}

A schematic of the experimental setup designed for studying the transmission of accelerated ions through capillaries has been reported in our paper [6] (Fig. 1 in this paper). This setup, which enters the implantation

\footnotetext{
* corresponding author; e-mail: KomarovF@bsu.by
}

complex formed on the basis of $1 \mathrm{MeV}$ electrostatic ion accelerator, consists of four units: (1) a system of ion beam formation, (2) a scattering chamber, (3) a measuring chamber, and (4) a system for detecting scattered ions. The parameters of a setup are as follows: the error in determining angles in measurements of angular distributions is not larger than $3.3 \times 10^{-3} \mathrm{deg}$ and error in the capillary orientation with respect to the beam axis is not larger than $2.5 \times 10^{-2} \mathrm{deg}$.

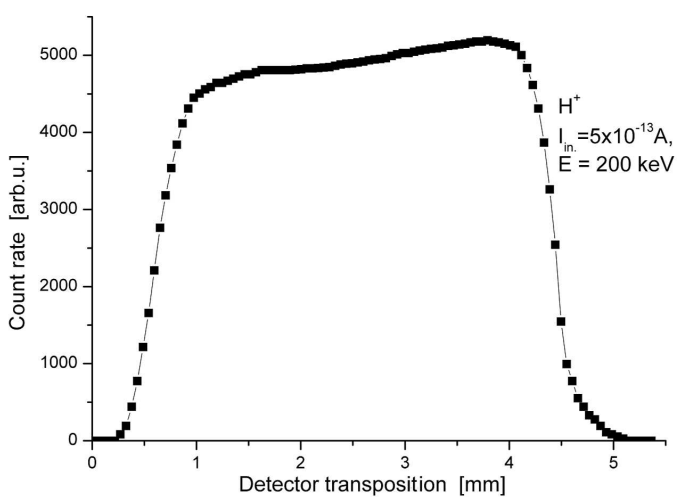

Fig. 1. Shape and angular distribution of a $200 \mathrm{keV}$ proton beam transmitted through the capillary.

The used proton beam energy of 150-300 keV was determined by a magnetic analyzer with an error of $\pm 0.1 \%$.

We measured the angular and the time distributions of protons transmitted through glass capillaries with an inlet and outlet diameters of 3.5 and $0.1 \mathrm{~mm}$, respectively, and the length of $150 \mathrm{~mm}$ in the range of taper angles of 0.5 to $2.2 \mathrm{deg}$. Input ion currents from $10^{-13}$ to $5 \times 10^{-10}$ A were used in these experiments. Angular distributions and energy spectra of transmitted protons were measured directly using a mobile silicon surface barrier detector positioned at a distance of $90 \mathrm{~cm}$ from a capillary holder. The total measured energy resolution of the recording system does not exceed $16 \mathrm{keV}$. 


\section{Experimental results and discussion}

The angular distribution of protons transmitted through the tapered capillary with an inlet diameter larger than an ion beam diameter and an outlet diameter of $100 \mu \mathrm{m}$ is shown in Fig. 1. It should be mentioned that a practically uniform distribution of ion beam density with sharp edges is registered in this case. Moreover, a spot size amounts to $3.8 \mathrm{~mm}$ that corresponds to a beam divergence of \pm 0.13 deg just as an initial beam divergence was $\pm 0.015 \mathrm{deg}$. An integral of an area under the curve in Fig. 1 shows that the fraction of transmitted protons is equal to $80 \%$, i.e. the number of transmitted ions relative to those entering into the capillary. Therefore, taking into account that the outlet diameter of the capillary is $100 \mu \mathrm{m}$ and the initial beam diameter amounts to $500 \mu \mathrm{m}$, the focusing factor reaches up to 20 .

An effort to measure angular distributions of transmitted protons at high currents at the input of the capillary failed as the measuring system functioning in a regime of individual particle registration was overloaded. Scanning of transmitted ion beams, at input beam currents of the order of $10^{-9} \mathrm{~A}$, showed that angular distributions of beams transmitted through the capillary are independent of the incident current.

In the case of this capillary oriented along the beam axis, the count rate versus the beam current at the input of the capillary is shown in Fig. 2. The results presented in Fig. 2 demonstrate a strong nonlinear behaviour of the current at the output of the capillary on intensity of the input beam up to an input current of $5 \times 10^{-13} \mathrm{~A}$. It is well accepted that such protons are guided electrostatically due to the charging up of the inner wall of capillaries made of insulating material [7].

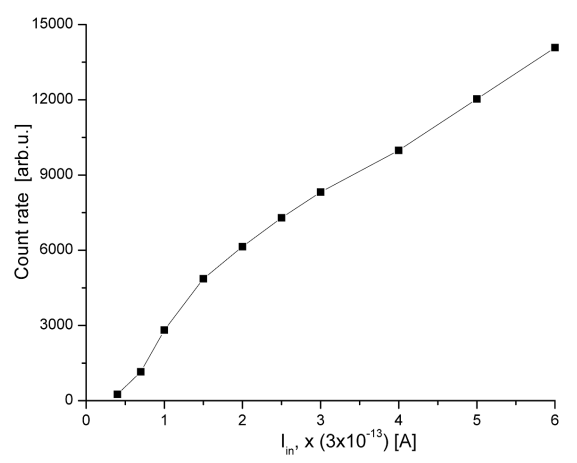

Fig. 2. Count rate of particles transmitted through the capillary versus proton current at the input of the capillary.

Figure 3 shows the time dependences for swift protons transmitted through cylindrical and tapered capillaries with a diameter of the inlet hole less than a diameter of the ion beam. In spite of the practically equal currents at the input of these capillaries, time evolutions of beam currents at the output of the capillaries are strongly different in shapes and frequencies of the beam intensity oscillations. Current pulse frequencies of ions transmitted
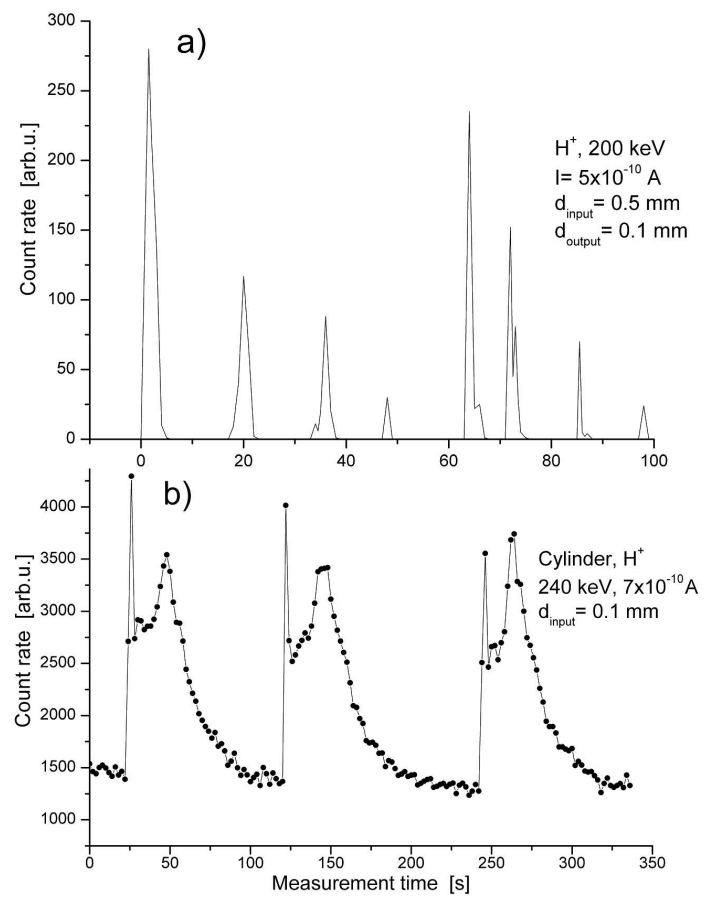

Fig. 3. Time distributions of protons transmitted through a tapered capillary (a) and cylindrical capillary (b).

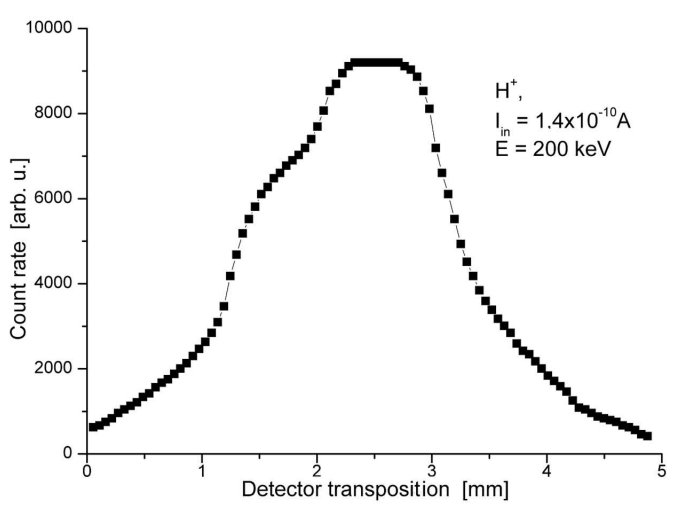

Fig. 4. Angular distribution of $200 \mathrm{keV}$ protons transmitted through a capillary with a taper angle of $0.5 \mathrm{deg}$.

through the tapered capillary exceed essentially those for the cylindrical capillary. On the contrary, shorter pulse durations are typical of the tapered capillary. It should be also noted that a pedestal at the level of $1300 \mathrm{pulse} / \mathrm{s}$ is observed in Fig. 3b just as in the case of the tapered capillary such an interpulse transparency is not revealed.

The above mentioned experimental results confirm our recent assumption [8] on a dominant role of charging up a face part of the capillary in the transformation of continuous ion beams into oscillating ones. This effect is not observed if an input hole of capillaries exceeds the beam diameter. A conducting protection of the face part of the tapered capillary (Fig. 3a) causes a total suppression of this effect (Fig. 4). The angular distribution of transmitted particles looks like the distribution presented in 


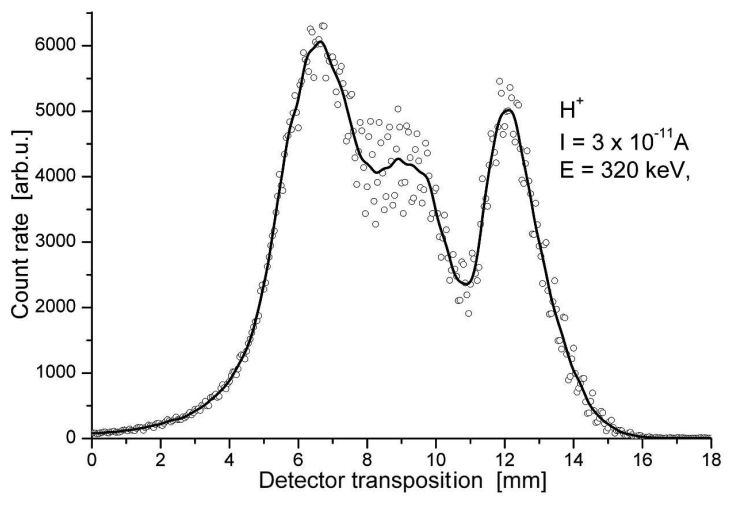

Fig. 5. Angular distribution of $320 \mathrm{keV}$ protons transmitted through a capillary with a taper angle of $2.2 \mathrm{deg}$.

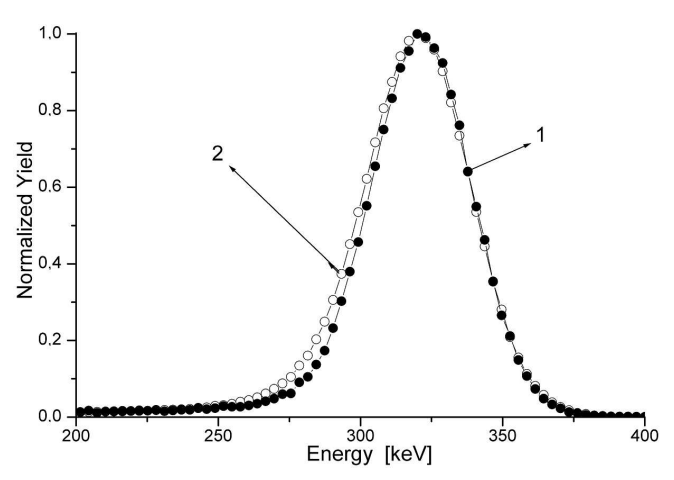

Fig. 6. Energy spectrum of transmitted protons with an initial energy of $320 \mathrm{keV}$ with and without capillary. The taper angle is $1.7 \mathrm{deg}$. Curve 1 is for the initial beam and curve 2 is for the transmitted beam.

Fig. 1. Nevertheless, the transparency of this capillary with a taper angle of $0.5 \mathrm{deg}$ is a few hundred times smaller than this parameter for the capillary with a taper angle of $1.7 \mathrm{deg}$. An analogous decrease of the transmission coefficient was observed in the capillary with a taper angle of $2.2 \mathrm{deg}$ (Fig. 5). At zero entrance angle (Fig. 5) three peaks are observed in the central part of the distribution. Such a behaviour has been revealed for the angular distribution of $240 \mathrm{keV}$ protons transmitted through the cylindrical capillary with a diameter of $0.5 \mathrm{~mm}$ and length of $178 \mathrm{~mm}$ (Fig. 3 in Ref. [6]).

The energy spectra of the initial and transmitted protons are shown in Fig. 6. Most of the ions transmit the capillary without energy loss, however, the low energy tail certainly exists (Fig. 6, curve 2). It means that those transmitted ions moving with higher transverse energies and suffering the small angle scattering lose their energy interacting with an inner surface of the capillary. It should be noted that these particles cause only very modest widening of the initial energy distribution (less than 5 to $6 \%$ ).

Generally speaking, the key to the understanding of these experimental observations is the notion of the two distinctly different time constants for the formed charge transport along the surface and within the bulk of capillaries. The interplay between these time constants is responsible for providing a dynamic equilibrium keeping up a Coulomb field large enough to deflect charged particles and small enough not to initiate a Coulomb blockade at the entrance of the capillary.

\section{Conclusions}

- We have confirmed that a few hundred keV proton beams are successfully focused by the tapered capillary optics.

- The areal density of the transmitted beam is enhanced by approximately 20 times.

- Charging up a face part of the capillary causes the transformation of continuous ion beams to oscillating ones.

- Most of the protons (94-95\%) in the energy range of 150 to $320 \mathrm{keV}$ transmit the capillary without energy loss.

- Changing a taper angle from $0.5 \mathrm{deg}$ to $1.7 \mathrm{deg}$ evidences the increase of the transmission coefficient more than by 300 times keeping the initial energy spectrum of ions.

- Compared with the conventional microbeam facilities, the usage of tapered capillaries is certainly simple and low-cost, thus providing an interesting technique of submicron Rutherford backscattering (RBS) or PIXE elemental analyses. Moreover, if the ion species are extended to heavier elements, the present method provides highly local versatile maskless ion implantation technique.

\section{References}

[1] T. Nebiki, T. Yamamoto, T. Narusawa, M.B.H. Breese, J.E. Teo, F. Watt, J. Vac. Sci. Technol. A 21, 1674 (2003).

[2] T. Nebiki, H. Robir, T. Narusawa, Nucl. Instrum. Methods Phys. Res. B 249, 226 (2006).

[3] J. Nasegawa, Sh. Shiba, H. Fukuda, Y. Oguri, Nucl. Instrum. Methods Phys. Res. B 266, 2125 (2008).

[4] R. Nakayama, M. Tona, N. Nakamura, H. Watanabe, N. Yoshiyasu, C. Yamada, A. Yamazaki, S. Ohtani, M. Sakurai, Nucl. Instrum. Methods Phys. Res. B 267, 2381 (2009).

[5] A. Razpet, G. Possnert, A. Johansson, A. Hallen, K. Hjort, Nucl. Instrum. Methods Phys. Res. B 222, 593 (2004).

[6] F.F. Komarov, A.S. Kamyshan, C. Karwat, Vacuum 83, 51 (2009).

[7] N. Stolterfoht, R. Hellhammer, J. Bundesmann, D. Fink, Nucl. Instrum. Methods Phys. Res. B 267, 226 (2009).

[8] A.S. Kamyshan, F.F. Komarov, J. Partyka, in: Proc. 8th Int. Conf. on Interaction of Radiation with Solids, Ed. V.M. Anishchik, BSU Publ. House, Minsk 2009, p. 32 . 